\title{
Metagenomic Next-Generation Sequencing for the Diagnosis of Pneumocystis jirovecii Pneumonia in Non-HIV-Infected Patients: A Retrospective Study
}

\author{
Juan Jiang · Lu Bai · Wei Yang · Wenzhong Peng · Jian An • \\ Yanhao Wu $\cdot$ Pinhua Pan · Yuanyuan Li
}

Received: April 28, 2021 / Accepted: June 10, 2021 / Published online: July 10, 2021

(C) The Author(s) 2021

\begin{abstract}
Introduction: This study aimed to evaluate the utility of metagenomic next-generation sequencing (mNGS) for the diagnosis of Pneumocystis jirovecii pneumonia (PJP) in non-human immunodeficiency virus-infected patients. Methods: We conducted a retrospective study. A total of 60 non-human immunodeficiency virus-infected PJP patients and 134 patients diagnosed with non-PJP pneumonia were
\end{abstract}

J. Jiang · L. Bai · W. Yang · W. Peng · J. An · Y. Wu · P. Pan $(\varangle) \cdot$ Y. Li $(\square)$

Department of Respiratory Medicine, National Key Clinical Specialty, Branch of National Clinical Research Center for Respiratory Disease, Xiangya Hospital, Central South University, Changsha 410008, People's Republic of China e-mail: pinhuapan668@hotmail.com

Y. Li

e-mail: leeround@csu.edu.cn

J. Jiang · L. Bai · W. Yang · W. Peng · J. An · Y. Wu · P. Pan . Y. Li

Hunan Provincial Clinical Research Center for Respiratory Diseases, Changsha, People's Republic of China

J. Jiang · L. Bai · W. Yang · W. Peng · J. An · Y. Wu . P. Pan . Y. Li

National Clinical Research Center for Geriatric Disorders, Xiangya Hospital, Changsha, People's Republic of China included. P. jirovecii and other co-pathogens identified by mNGS in bronchoalveolar lavage fluid and/or blood samples were analyzed. Using clinical composite diagnosis as the reference standard, we compared the diagnostic performance of mNGS in PJP with conventional methods, including Gomori methenamine silver staining and serum (1,3)- $\beta$-D-glucan. Modifications of antimicrobial treatment for PJP patients after the report of mNGS results were also reviewed.

Results: mNGS reached a sensitivity of $100 \%$ in diagnosing PJP, which was remarkably higher than Gomori methenamine silver staining (25.0\%) and serum (1,3)- $\beta$-D-glucan (67.4\%). The specificity of $\mathrm{mNGS}(96.3 \%)$ significantly surpassed serum (1,3)- $\beta$-D-glucan (81.4\%). Simultaneous mNGS of bronchoalveolar lavage fluid and blood samples was performed in 21 out of 60 PJP patients, and it showed a concordance rate of $100 \%$ in detecting $P$. jirovecii. Besides, mNGS showed good performance in identifying co-pathogens of PJP patients, among which cytomegalovirus and Epstein-Barr virus were most commonly seen. Initial antimicrobial treatment was modified in $71.7 \%$ of PJP patients after the report of mNGS results. Conclusion: mNGS is a useful diagnostic tool with good performance for the diagnosis of PJP and the detection of co-pathogens. mNGS of bronchoalveolar lavage fluid and/or blood samples is suggested in patients with presumptive diagnosis of PJP. Blood samples may be a 
good alternative to bronchoalveolar lavage fluid for mNGS when bronchoscopic examination is not feasible.

Keywords: Diagnosis; Metagenomics; Nextgeneration sequencing; Pneumocystis jirovecii; Pneumocystis jirovecii pneumonia

\section{Key Summary Bullet Points}

Metagenomic next-generation sequencing achieved a sensitivity of $100 \%$ and a specificity of $96.3 \%$ for the diagnosis of Pneumocystis jirovecii pneumonia.

Simultaneous metagenomic nextgeneration sequencing of bronchoalveolar lavage fluid and blood samples showed a good concordance rate in Pneumocystis jirovecii pneumonia patients.

Metagenomic next-generation sequencing was advantageous to identify copathogens in Pneumocystis jirovecii pneumonia patients with mixed infections.

\section{DIGITAL FEATURES}

This article is published with digital features, including a summary slide, to facilitate understanding of the article. To view digital features for this article go to https://doi.org/10.6084/ m9.figshare.14763081.

\section{INTRODUCTION}

Pneumocystis jirovecii pneumonia (PJP) is a common opportunistic infection in the immunocompromised population. It is caused by infection of Pneumocystis jirovecii (P. jirovecii) and remains a major threat in both human immunodeficiency virus (HIV) and non-HIV-infected patients. While $70-90 \%$ of HIV-infected patients undergo PJP for once or more, HIV-negative patients who are immunocompromised for other reasons are also at high risk of PJP and usually have a more progressive course and poorer prognosis than the HIV-infected population [1]. Recently, the prevalence of PJP in the non-HIV population has been increasing and drawn intense attention [2].

Rapid and accurate diagnosis is critical for clinical prognosis of PJP patients. Clinical manifestations of PJP patients are non-specific, including fever, cough and progressive hypoxia. To date, $P$. jirovecii cannot be reliably grown in vitro. The definite diagnosis of PJP can be made only when the trophic cells and/or the asci are observed under the microscope in respiratory tract specimens after conventional or immunofluorescence staining. However, the sensitivity of conventional staining is low, and a negative staining report is far from enough to exclude the diagnosis of PJP [3], while immunofluorescence staining is not routinely performed in many hospitals. Currently, the polymerase chain reaction (PCR) method has been widely used for detecting $P$. jirovecii because of its good sensitivity (94-99\%) and specificity (89-93\%) even in low-burden specimens [4]. However, its diagnostic value is limited in identifying mixed infections, which is common in immunocompromised PJP patients. In addition, (1,3)- $\beta$-D-glucan (BDG) is a sensitive but unspecific serologic biomarker of PJP [5]. Compared to HIV-infected patients, diagnosis of PJP in the non-HIV immunocompromised population is more difficult. It has been reported that PJP patients without AIDS have fewer Pneumocystis organisms in the lung than those with AIDS, potentially reducing the detection rate of $\mathrm{PJ}$ in respiratory tract specimens [6]. Besides, while HIV-infected patients generally present bilateral interstitial and alveolar infiltrates in perihilar areas, radiologic features of non-HIV-infected PJP patients are more variable [2], which also increases the complexity of diagnosis. Therefore, there is an urgent need for exploring more accurate and efficient microbiologic diagnostic tools for PJP in the HIV-negative population.

Recently, metagenomic next-generation sequencing (mNGS) has been considered a promising microbial identification technology in infectious diseases [7]. Based on highthroughput sequencing, mNGS detects 
microbial nucleic acids in a variety of specimens and identifies a wide range of pathogens in a single run [8]. mNGS is unbiased, rapid and highly sensitive, making it a popular microbial test in infectious diseases, especially for pathogens unidentifiable by routine diagnostic methods [9]. Previously, the application of mNGS has been reported in the diagnosis of PJP in several case reports [10-12]. However, the utility of mNGS for the diagnosis of PJP in nonHIV-infected patients remains largely unexplored.

Therefore, we evaluated the diagnostic performance of mNGS in non-HIV-infected patients with PJP in this retrospective study.

\section{METHODS}

\section{Study Design and Subjects}

In this retrospective study, we consecutively enrolled 60 PJP patients who were admitted to the Department of Respiratory Medicine of Xiangya Hospital, Central South University (Changsha, China), from 1 July 2018 to 30 November 2020. Patients were eligible for enrollment if they met all the following criteria: (1) immunocompromised conditions, including but not limited to hematologic malignancies, solid tumors, rheumatic diseases, long-term systemic use of corticosteroids ( $>0.3 \mathrm{mg} / \mathrm{kg}$ /day of prednisone equivalent for $\geq 3$ weeks), use of immunosuppressive agents (including chemotherapeutic agents for malignancies, but not corticosteroids), solid organ transplantation and hematopoietic stem cell transplantation; (2) typical clinical manifestations of PJP, including fever, cough, dyspnea, and progressive hypoxemia; (3) radiologic findings suggestive of PJP in bilateral lungs that newly emerged on chest computed tomography (CT); (4) bronchoalveolar lavage fluid (BALF) and/or blood samples were collected for mNGS. In this study, microbiologic tests for $P$. jirovecii included $\mathrm{mNGS}$ and direct microscopic examination after Gomori methenamine silver (GMS) staining. Meanwhile, 134 patients who were admitted to our department because of pulmonary infections and subsequently confirmed as having non-PJP pneumonia during the same time interval were consecutively enrolled as control. The clinical composite diagnosis of PJP or non-PJP pneumonia was made by two senior expert pulmonologists (LY and PP) after discussion with the healthcare team, based on clinical symptoms, laboratory findings, chest radiology, microbiologic tests and treatment response. Patients were excluded if they met any of the following criteria: (1) age $<18$ years old; (2) HIV infection; (3) mNGS was not performed; (4) medical record was incomplete.

\section{Bronchoalveolar Lavage}

Bronchoalveolar lavage was performed by experienced bronchoscopists after local anesthesia with lidocaine in accordance with the standard procedures at Xiangya Hospital. Briefly, the sampling location was selected based on chest CT images. Three $20-\mathrm{ml}$ fractions of sterile saline were instilled into the target subsegmental bronchi. BALF was retrieved by gentle syringe suction, put into sterile containers and immediately send for mNGS test and GMS staining. The first $20 \mathrm{ml}$ was usually discharged to avoid contamination, whereas the remaining samples were collected for detection.

\section{Sample Processing and DNA Extraction for $\mathrm{mNGS}$}

Briefly, $600 \mu \mathrm{l}$ of BALF was taken from patients and mixed with lysozyme and $1 \mathrm{~g} 0.5-\mathrm{mm}$ glass beads, and then the mixture was attached to a horizontal platform on a vortex mixer and agitated vigorously at $2800-3200 \mathrm{rpm}$ for $30 \mathrm{~min}$. For nucleic acid extraction, $300 \mu$ l of supernatant was transferred to a $1.5-\mathrm{ml}$ centrifuge tube. Volumes of $3-5 \mathrm{~m}$ of patients' blood were centrifuged at $1600 \mathrm{~g}$ for $10 \mathrm{~min}$ at $4{ }^{\circ} \mathrm{C}$ for plasma separation. For DNA extraction, $300 \mu \mathrm{l}$ of plasma was transferred to a 2-ml sterile tube. DNA was extracted using the TIANamp Micro DNA kit (Tiangen Biotech) according to standard procedures [13]. 


\section{Construction of DNA Library and Sequencing}

The DNA library was constructed by DNA fragmentation, end repair and PCR amplification using MGIEasy Cell-free DNA Library Prep Set (MGI Tech). Agilent 2100 (Agilent Technologies) and Qubit 2.0 (Invitrogen) were used for library quality control. The double-stranded DNA library was converted into single-stranded circular DNA using DNA degradation and circularization. The DNA Nanoballs were generated by rolling circle amplification technology. Qualified DNA Nanoballs were loaded on the chip and then performed $20 \mathrm{M}$ 50-bp single-end sequencing on the MGISEQ-2000 sequencing platform (BGI Genomics).

\section{Bioinformatic Analysis}

Bioinformatics analysis was performed on samples with raw data $>10$ million. By removing low-quality [14], short reads (length $<35 \mathrm{bp}$ ), the human host sequence mapped to the human reference genome (hg19) using BurrowsWheeler alignment [15] and low complexity reads. The high-quality data were classified by simultaneously aligned to four Microbial Genome Databases, including viruses, bacteria, fungi and parasites. The databases were downloaded from NCBI (ftp:/ftp.ncbi.nlm.nih.gov/ genomes/). It contains 1798 whole-genome sequences of DNA viral taxa, 6350 bacterial genomes or scaffolds, 1064 fungi related to human infection and 234 parasites associated with human diseases. The coverage ratio and depth of each microorganism were calculated using BEDTools [16]. Whole-blood samples from healthy donors as a negative control used the same protocol as each batch sample.

Clinically significant microbes were defined as described in the previous study [17]. For bacteria (excluding mycobacteria), fungi (excluding molds), viruses, parasites and other atypical pathogens, a microbe was considered clinically significant when its relative abundance at the species level was $>30 \%$, and its pulmonary pathogenicity has been proven in the literature. Mycobacterium detected by
mNGS was considered clinically significant when the stringently mapped read number $($ SMRN) at the species level was $>3$. Molds with literature-proven pulmonary pathogenicity were considered clinically significant microbes when the SMRN at the species level was $>10$. Oral commensals were not considered clinically significant microbes regardless of their abundance. Subsequently, clinically significant microbes were classified as putative co-pathogens if patients showed suggestive symptoms, laboratory findings and/or radiologic abnormalities.

\section{Statistical Analysis}

SPSS 25.0 software (IBM Corp., Armonk, NY, USA) was used for statistical analysis. Continuous variables are presented as medians and interquartile ranges and categorical variables as counts and percentages. The Mann-Whitney $U$ test was used for comparing the differences of continuous variables between PJP and non-PJP groups and the chi-square test for categorical variables. Spearman's test was performed to analyze correlation between the SMRN of $P$. jirovecii detected by $\mathrm{mNGS}$ and serum BDG levels. Sensitivity, specificity, positive predict value (PPV) and negative predict value (NPV) were calculated using the clinical composite diagnosis as the reference standard; 95\% confidence intervals for these proportions were determined using Wilson's method. Significance was fixed at $P<0.05$.

\section{Ethics Statement}

This study was approved by the Institutional Review Board and Ethics Committee of Central South University and conducted according to the Declaration of Helsinki. The ethics committee approved the waiver of informed consent owing to the retrospective nature of the review. All research data were de-identified and anonymously analyzed. 
Table 1 Clinical characteristics, laboratory findings and radiologic features of PJP and non-PJP patients on admission

\begin{tabular}{|c|c|c|c|}
\hline $\begin{array}{l}\text { Characteristics (median } \\
{[\mathrm{IQR}] \text { or } n[\%] \text { ) }}\end{array}$ & $\begin{array}{l}\text { PJP patients } \\
(n=60)\end{array}$ & $\begin{array}{l}\text { Non-PJP patients } \\
(n=134)\end{array}$ & $P$ value \\
\hline Age (years) & $53(44-61)$ & $59(53-67)$ & 0.019 \\
\hline Male & $37(61.7)$ & $92(68.7)$ & 0.34 \\
\hline \multicolumn{4}{|l|}{ Clinical symptoms } \\
\hline Fever & $43(71.7)$ & $90(67.2)$ & 0.532 \\
\hline Cough & $39(65.0)$ & $113(84.3)$ & 0.003 \\
\hline Expectoration & $32(53.3)$ & $98(73.1)$ & 0.007 \\
\hline Dyspnea & $49(81.7)$ & $95(70.9)$ & 0.113 \\
\hline Hemoptysis & $5(8.3)$ & $10(7.5)$ & 0.834 \\
\hline Chest pain & $4(6.7)$ & $6(4.5)$ & 0.524 \\
\hline \multicolumn{4}{|l|}{ Immunocompromised conditions } \\
\hline Systemic use of corticosteroids & $43(71.7)$ & $24(17.9)$ & $<0.001$ \\
\hline Use of immunosuppressive agents & $39(65.0)$ & $15(11.2)$ & $<0.001$ \\
\hline Hematologic malignancies & $10(16.7)$ & $1(0.7)$ & $<0.001$ \\
\hline Solid tumors & $4(6.7)$ & $6(4.5)$ & 0.524 \\
\hline Rheumatic diseases & $22(36.7)$ & $25(18.7)$ & 0.007 \\
\hline Solid organ transplantation & $3(5.0)$ & $0(0)$ & 0.009 \\
\hline HSC transplantation & $3(5.0)$ & $0(0)$ & 0.009 \\
\hline $\mathrm{PaO}_{2} / \mathrm{FiO}_{2}(\mathrm{mmHg})$ & $143(105-181)$ & $155(117-280)$ & 0.35 \\
\hline White blood cells $\left(\times 10^{9} / 1\right)$ & $7.4(5.3-10.9)$ & $11.8(8.0-16.4)$ & 0.123 \\
\hline Neutrophils $\left(\times 10^{9} / 1\right)$ & $6.6(4.5-9.7)$ & $10.9(6.6-15.5)$ & 0.115 \\
\hline Lymphocytes $\left(\times 10^{9} / \mathrm{l}\right)$ & $0.5(0.3-0.8)$ & $0.5(0.4-0.9)$ & 0.648 \\
\hline Hemoglobin $(\mathrm{g} / \mathrm{l})$ & $98(83-117)$ & $109(88-122)$ & 0.026 \\
\hline Platelet $\left(\times 10^{9} / \mathrm{l}\right)$ & $147(71-215)$ & $198(130-265)$ & 0.068 \\
\hline \multicolumn{4}{|l|}{ Serum BDG (ng/l) } \\
\hline$\geq 80$ & $31(67.4[\mathrm{n}=46])$ & $16(18.6[\mathrm{n}=86])$ & $<0.001$ \\
\hline$\geq 500$ & $13(28.3[n=46])$ & $1(1.2[\mathrm{n}=86])$ & $<0.001$ \\
\hline $\mathrm{LDH}(\mathrm{U} / \mathrm{l})$ & $496(338-736)$ & $457(266-702)$ & 0.231 \\
\hline CRP (mg/l) & $88.3(35.8-159.0)$ & $138.0(33.2-217.0)$ & 0.614 \\
\hline PCT (ng/ml) & $0.30(0.09-1.88)$ & $0.76(0.24-2.76)$ & 0.138 \\
\hline \multicolumn{4}{|l|}{ Chest CT images } \\
\hline Ground-glass opacity & $41(68.3)$ & $40(29.9)$ & $<0.001$ \\
\hline Patchy shadowing & $45(75.0)$ & $81(60.4)$ & 0.05 \\
\hline
\end{tabular}


Table 1 continued

\begin{tabular}{lllr}
\hline $\begin{array}{l}\text { Characteristics (median } \\
[\mathbf{I Q R}] \text { or } \boldsymbol{n}[\%])\end{array}$ & $\begin{array}{l}\text { PJP patients } \\
(\boldsymbol{n}=\mathbf{6 0})\end{array}$ & $\begin{array}{l}\text { Non-PJP patients } \\
(\boldsymbol{n}=\mathbf{1 3 4})\end{array}$ & $\boldsymbol{P}$ value \\
\hline Interstitial patterns & $20(33.3)$ & $41(30.6)$ & 0.704 \\
Consolidation & $20(33.3)$ & $46(34.3)$ & 0.892 \\
Pleural effusion & $8(13.3)$ & $64(47.8)$ & $<0.001$ \\
Cystic changes & $3(5.0)$ & $7(5.2)$ & 0.948 \\
\hline
\end{tabular}

$I Q R$ interquartile range, $\mathrm{HSC}$ hematopoietic stem cells, $\mathrm{PaO}_{2}$ arterial partial pressure of oxygen, $\mathrm{FiO}_{2}$ fraction of inspired oxygen, $B D G$ (1,3)- $\beta$-D-glucan, $L D H$ lactate dehydrogenase, $C R P$ C-reactive protein, $P C T$ procalcitonin, $C T$ computed tomography

\section{RESULTS}

\section{Clinical Characteristics, Laboratory Findings and Radiologic Features of Non- HIV-Infected PJP Patients}

Patient characteristics on admission are summarized in Table 1. A total of 60 non-HIV-infected PJP and 134 non-PJP patients were included in this study. The median ages (53 vs. 59 years old) and gender compositions of these two cohorts were similar. The most common symptoms of PJP patients included dyspnea $(81.7 \%)$, fever $(71.7 \%)$ and cough (65.0\%). Compared to those with non-PJP pneumonia, cough and expectoration were less frequently seen in PJP patients. Rheumatic disease (36.7\%) ranked first, and hematologic malignancy (16.7\%) ranked second among the underlying diseases of PJP. As expected, PJP patients had various immunosuppressive conditions; $71.7 \%$ of them received long-term systemic corticosteroids, $65.0 \%$ received immunosuppressive agents. In contrast, these immunosuppressive conditions were far less common in the non-PJP cohort.

The median $\mathrm{PaO}_{2} / \mathrm{FiO}_{2}$ was $143 \mathrm{mmHg}$ in PJP patients, suggesting serious hypoxemia. The white blood cell count, neutrophil count and serum procalcitonin level of PJP patients appeared lower than in non-PJP ones, but no significance was observed. All PJP patients showed lymphopenia with a median lymphocyte count of $0.5 \times 10^{9} / 1$ in peripheral blood and had significantly lower hemoglobin levels
(98 vs. $109, P=0.026)$ than the non-PJP cohort. Elevated serum level of BDG $(\geq 80 \mathrm{ng} / \mathrm{l})$ was observed in $67.4 \%$ of PJP and $18.6 \%$ of non-PJP patients $(P<0.001)$, respectively. Median serum levels of lactate dehydrogenase (496 vs. $457 \mathrm{U} / \mathrm{l}$ ) and C-reactive protein (88.3 vs. $138.0 \mathrm{mg} / \mathrm{l}$ ) were remarkably increased in both cohorts. Among PJP patients, the most frequently seen radiologic findings on chest CT were ground-glass opacity (68.3\%) and patchy shadowing $(75.0 \%)$. Pleural effusion was significantly less common than in non-PJP patients (13.3\% vs. $47.8 \%, P<0.001)$.

\section{Comparison of Diagnostic Performance Among mNGS, GMS Staining and Serum BDG in PJP}

GMS staining is a classical method for microbiologic diagnosis of PJP, and BDG is a widely used serologic biomarker of PJP. Therefore, we compared the diagnostic performance of $\mathrm{mNGS}$ with GMS staining and serum BDG in 60 PJP patients. As shown in Table 2, mNGS of BALF and/or blood samples was performed in all patients, GMS staining of BALF in 60 patients (28 in PJP and 32 in non-PJP cohort) and serum BDG in 132 patients (46 in PJP and 86 in nonPJP cohort). Herein, diagnostic sensitivity and specificity of mNGS were calculated using the clinical composite diagnosis as reference standard. The sensitivity of mNGS was 100\% (95\% CI, 94.0-100\%), which was markedly higher than that of GMS staining [25.0\% $(95 \%$ CI $10.7-44.9 \%), P<0.001]$ and BDG [67.4\% (95\% 
Table 2 Diagnostic performance of mNGS, GMS staining and serum BDG in non-HIV-infected PJP patients

\begin{tabular}{|c|c|c|c|c|c|c|}
\hline Methods & $\begin{array}{l}\text { PJP } \\
\text { cohort }\end{array}$ & $\begin{array}{l}\text { Non-PJP } \\
\text { cohort }\end{array}$ & $\begin{array}{l}\text { Sensitivity (95\% } \\
\text { CI) }\end{array}$ & $\begin{array}{l}\text { Specificity (95\% } \\
\text { CI) }\end{array}$ & $\begin{array}{l}\text { PPV (95\% } \\
\text { CI) }\end{array}$ & $\begin{array}{l}\text { NPV }(95 \% \\
\text { CI })\end{array}$ \\
\hline mNGS + & 60 & 5 & $100 \%$ & $96.3 \%$ & $92.3 \%$ & $100 \%$ \\
\hline- & 0 & 129 & $(94.0-100)$ & (91.5-98.8) & $(83.0-97.5)$ & $(97.2-100)$ \\
\hline $\begin{array}{l}\text { GMS } \\
\text { staining + }\end{array}$ & 7 & 0 & $25.0 \%^{* * *}$ & $100 \%$ & $100 \%$ & $60.4 \%^{* * *}$ \\
\hline- & 21 & 32 & $(10.7-44.9)$ & $(89.1-100)$ & $(59.0-100)$ & $(46.0-73.5)$ \\
\hline $\begin{array}{l}\text { Serum } \\
\qquad \mathrm{BDG}+\end{array}$ & 31 & 16 & $67.4 \%^{\# \# \#}$ & $81.4 \%{ }^{\# \# \#}$ & $66.0 \%{ }^{\# \# \#}$ & $82.6 \% \%^{\# \# \#}$ \\
\hline- & 15 & 70 & $(52.0-80.5)$ & $(71.6-89.0)$ & $(50.7-79.1)$ & $(72.6-89.8)$ \\
\hline
\end{tabular}

GMS staining Gomori methenamine silver staining, $B D G(1,3)-\beta$-D-glucan, serum BDG $\geq 80 \mathrm{ng} / \mathrm{l}$ was defined as positive, $C I$ confidence intervals, $P P V$ positive predict value, $N P V$ negative predict value

${ }^{* * *} P<0.001$ when comparing GMS staining with mNGS

\#\#\# $P<0.001$ when comparing serum BDG with mNGS

CI $52.0-80.5 \%), \quad P<0.001] . \quad$ Furthermore, mNGS showed a good specificity of $96.3 \%(95 \%$ CI 91.5-98.8\%), which was comparable to GMS staining [100\% (95\% CI 89.1-100\%)] and significantly higher than serum BDG $[81.4 \%(95 \%$ CI 71.6-89.0\%)]. As a result, mNGS reached a PPV of $92.3 \%$ and a NPV of $100 \%$, which surpassed both GMS staining and BDG. Besides, the SMRNs of $P$. jirovecii detected by $\mathrm{mNGS}$ in BALF $(R=0.421, P=0.004)$ and blood samples

A

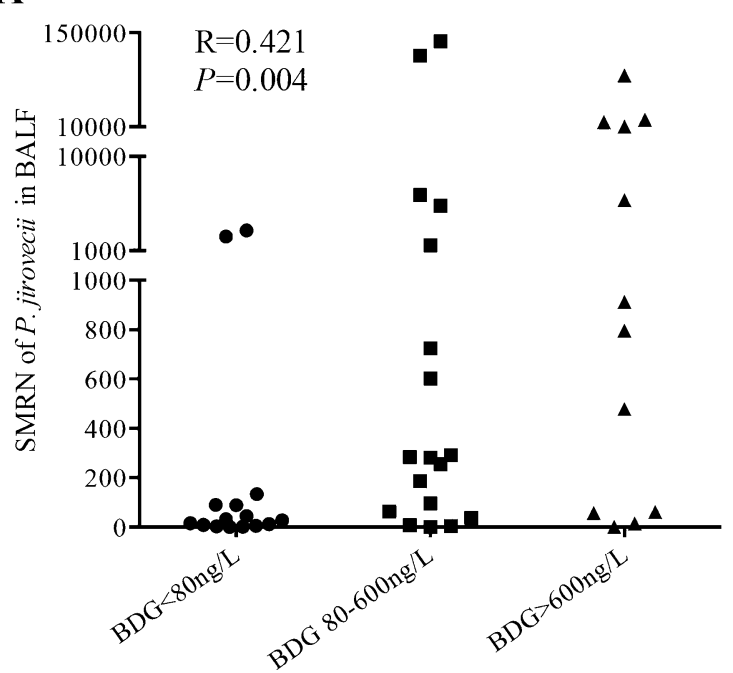

( $R=0.432, P=0.050)$ were positively correlated with serum BDG levels (Fig. 1).

\section{Detection of $P$. jirovecii in BALF and/ or Blood Samples by mNGS}

In this study, mNGS of BALF samples was performed in 53 PJP patients and mNGS of blood samples in 28 PJP patients. Among them, 32 had sole mNGS of the BALF sample, 7 had sole

B

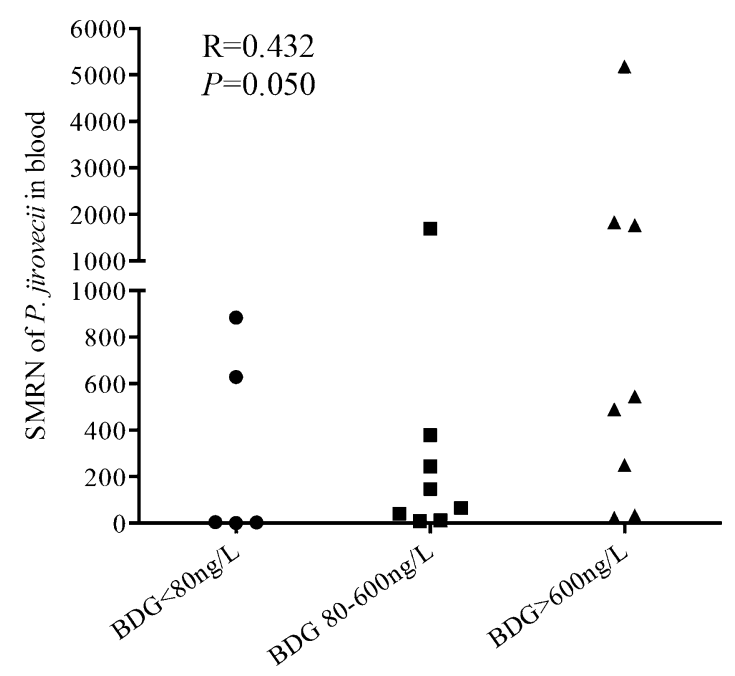

Fig. 1 Correlation analysis between the SMRN of $P$. jirovecii detected by mNGS and serum BDG levels. A mNGS of BALF samples; B mNGS of blood samples 


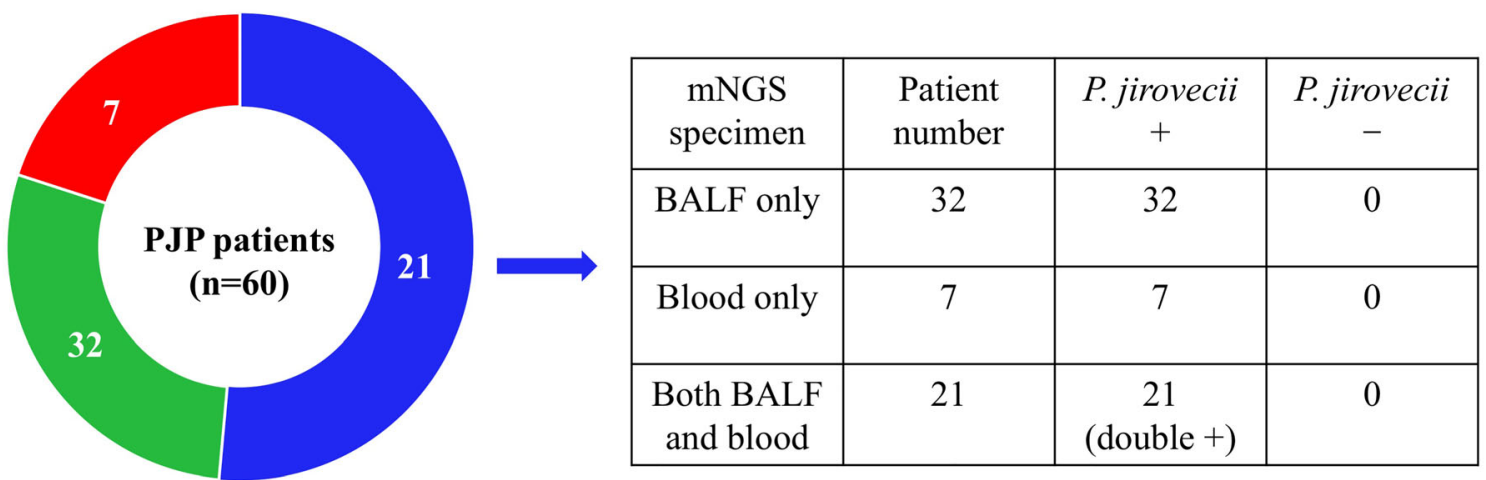

BALF $\square$ Blood $\square$ BALF and blood

Fig. 2 Detection of $P$. jirovecii by mNGS in BALF and blood samples obtained from PJP patients

mNGS of the blood sample, and 21 had both (Fig. 2). Remarkably, P. jirovecii was detected by mNGS in all the matched BALF and blood samples, reaching a concordance rate of $100 \%$.

\section{Mixed Infections and Co-pathogens Detected by mNGS}

Mixed infections are common in PJP patients because of incomplete immunity. Numbers of cases with putative mixed infections detected by mNGS are summarized in Fig. 3. Viruses and bacteria were the most common co-pathogens observed in PJP patients. Both P. jirovecii-virus coinfection and $P$. jirovecii-virus-bacteria coinfection were identified by mNGS in 15 (25.0\%) of 60 patients (Fig. 3A). Top five copathogens detected by mNGS included cytomegalovirus, Epstein-Barr virus, human herpesvirus 1, Candida and Torque teno virus (Fig. 3B). Of note, Mycobacterium tuberculosis was detected in seven PJP patients, indicating that more attention should be paid to mixed infections of PJP and tuberculosis.

\section{Impact of mNGS on Antimicrobial Treatment of PJP Patients}

Records of antimicrobial treatment which targeted $P$. jirovecii and other pathogens during
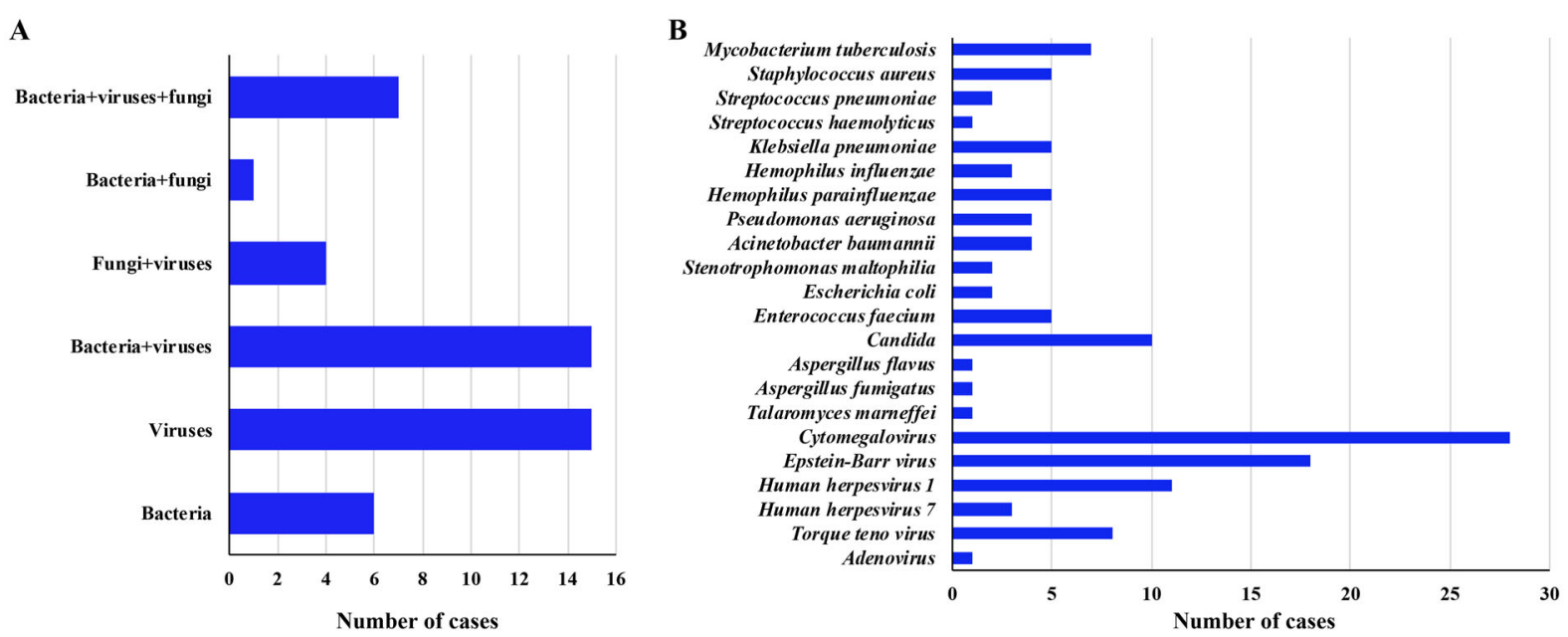

Fig. 3 Mixed infections and co-pathogens identified by mNGS in 60 PJP patients. A number of PJP patients with mixed infections; $\mathbf{B}$ number of PJP patients infected with various co-pathogens 
Table 3 Impact of mNGS on antimicrobial treatment on PJP patients

\begin{tabular}{ll}
\hline Modifications $(\boldsymbol{n}[\%])$ & PJP patients $(\boldsymbol{n}=\mathbf{6 0})$ \\
\hline Remove 1 agent & $10(16.7)$ \\
Remove 2 agents & $3(5.0)$ \\
Reduce antimicrobial spectrum & $14(23.3)$ \\
Add 1 agent & $13(21.7)$ \\
Add 2 agents & $3(5.0)$ \\
Add TMP-SMZ & $22(36.7)$ \\
Add caspofungin & $11(18.3)$ \\
No change & $17(28.3)$ \\
\hline
\end{tabular}

TMP-SMZ trimethoprim-sulfamethoxazole; remove 1 (or 2) agent, the number of antimicrobial agent types reduced by 1 (or 2) after the report of mNGS results; add 1 (or 2) agent, the number of antimicrobial agent types reduced by 1 (or 2) after the report of mNGS results

hospitalization were retrieved from 60 PJP patients. Based on the mNGS results, initial antimicrobial treatment was modified in $71.7 \%$ of patients; $21.7 \%$ of patients had one or two antimicrobial agents removed, $23.3 \%$ had the antimicrobial spectrum reduced, and $26.7 \%$ had one or two antimicrobial agents added (Table 3); $36.7 \%$ of patients did not receive trimethoprim-sulfamethoxazole (TMP-SMZ) of therapeutic dose until the report of mNGS results.

\section{DISCUSSION}

Herein, we conducted a retrospective study to evaluate the diagnostic performance of mNGS in HIV-negative PJP patients. We found that mNGS had an excellent sensitivity and specificity in the diagnosis of PJP and was advantageous to identify co-pathogens in pulmonary mixed infections. Furthermore, mNGS of blood and BALF samples showed a good concordance in the detection of $P$. jirovecii.

PJP is a common opportunistic infection which can be life-threatening in immunocompromised hosts. Currently, there is a growing incidence of PJP in the non-HIV immunocompromised population due to the increasing prevalence of immunosuppressive conditions, such as hematologic malignancies, solid tumors, systemic corticosteroid therapy, immunosuppressive therapy and organ transplantation [18]. In the non-HIV-infected population, PJP usually progresses to respiratory failure within a short time and causes a mortality rate of $30-60 \%$, which was significantly higher than in the HIV-infected population [2]. Since effective anti-PJP treatment regimens have been clearly defined, and TMP-SMZ is recommended as the first-line antimicrobial treatment [19], microbiologic diagnosis of PJP is the "rate-limiting" step to achieve prompt treatment and improve prognosis of patients. So far, conventional staining, PCR and serum BDG are widely used for the microbiologic diagnosis of PJP. However, these methods have their own limitations. Conventional staining requires a heavy pathogen burden in the lung and experienced microbiologists to ensure the detection rate of $P$. jirovecii under the microscope; thus, it can be insensitive and biased. PCR has been reported to be highly sensitive and specific in detecting $P$. jirovecii, but it delivers little value to identify mixed infections by a single run, especially for those rare or novel pathogens. Additionally, the non-specificity of BDG limits its application in the diagnosis of PJP.

$\mathrm{mNGS}$ is a microbiologic diagnostic tool that detects all nucleic acids of microorganisms whose sequencing data were included in the database library and allows for an unbiased approach to the detection of pathogens [20]. Diagnostic performance of mNGS has been highlighted in infections of the respiratory tract [21, 22], blood stream [23], central nervous system [24] and prosthetic joints [25]. Data from recent studies supported its advantages in detecting opportunistic pathogens and mixed infections, especially in detecting uncultivable pathogens $[26,27]$. mNGS is highly sensitive to identifying a very small amount of pathogens, but its diagnostic performance in PJP remains unclear. In the present study, our data revealed the sensitivity of mNGS was $100 \%$ in non-HIVinfected PJP patients, which was dramatically higher than GMS staining and serum BDG. 
Furthermore, mNGS showed a good specificity of $96.3 \%$, which was comparable to GMS staining and significantly better than serum BDG. These results suggest that early application of mNGS can facilitate the rapid and accurate diagnosis of PJP.

Another distinct advantage of mNGS over other microbiologic tests is the broad spectrum of pathogen identification, which allows the detection of mixed infections by a single run in PJP patients. Therefore, patients with mixed infections may be further benefited by this technique. One tricky problem of interpreting mNGS results is the lack of a standard method and widely accepted threshold values. It is not easy to decide whether microbes detected by mNGS are clinically significant pathogens or colonized microbes. So far, the SMRN and abundance score of microbes have been frequently used parameters among different sequencing platforms. Clinically significant pathogens must be classified based on comprehensive analysis of these parameters, clinical characteristics and laboratory findings. Our data showed that mixed infectious were detected in $80 \%$ of PJP patients, among which viral and bacterial mixed infections were the most common. The unbiased broad-spectrum detection of mNGS would further provide guidance for effective antimicrobial treatment. In the present study, initial antimicrobial treatments were modified for $71.7 \%$ of patients after the report of mNGS results. More importantly, $36.7 \%$ of PJP patients did not receive TMP/SMZ and $18.3 \%$ did not receive caspofungin until the report of mNGS results, demonstrating that mNGS is useful for the diagnosis and proper treatment of PJP.

It is worth noting that simultaneous mNGS of BALF and blood samples obtained from the same patient showed highly consistent results in detection of $P$. jirovecii. In this study, a total of seven patients who either could not tolerate or declined bronchoscope examination were diagnosed with PJP through mNGS of blood samples. These encouraging results strongly indicated that mNGS of blood samples could be a good alternative for patients with presumptive diagnosis of PJP when bronchoscopic examination was not doable. Furthermore, mNGS of blood samples may be helpful in distinguishing $P$. jirovecii colonization from infection. It is well acknowledged that $P$. jirovecii resides in respiratory tracts of healthy humans, so the interpretation of mNGS results of respiratory tract specimens can be sometimes difficult, especially when the SMRN of $P$. jirovecii is small. In this condition, $P$. jirovecii identified by $\mathrm{mNGS}$ in blood samples could be more convincing because it should not exist in blood stream of healthy humans [28]. Therefore, on the basis of our data, we propose that BALF and blood samples should be simultaneously collected for mNGS when the diagnosis of PJP is considered.

This study has limitations. First, it is a singlecenter retrospective study; thus, intrinsic bias was unavoidable. Second, diagnostic performances of $\mathrm{mNGS}$ and PCR were not compared in this study because PCR of $P$. jirovecii was not routinely performed in our hospital. This line of query should be explored in future work. Finally, mNGS has drawbacks while it offers numerous advantages. It is difficult to distinguish $P$. jirovecii colonization from infection because there are no widely accepted quantitative cutoffs or threshold values for mNGS. Therefore, the definite diagnosis of PJP must be based on the comprehensive analysis of clinical characteristics, laboratory abnormalities, radiologic findings and microbiologic proofs rather than mNGS alone. Additionally, a single run of mNGS costs 500-600 dollars and takes $24-48 \mathrm{~h}$ to finish all procedures, which may limit its application in clinical practice. To evaluate the diagnostic value of mNGS in PJP, comparative analysis between PCR and mNGS must be done, and more studies are needed to explore potential cut-off or threshold values for differentiating $P$. jirovecii infection from colonization. Dynamic testing of mNGS in PJP patients during the whole course of illness is also of interest to investigate its potential utility in predicting the efficacy of anti-PJP treatment and clinical outcomes.

\section{CONCLUSIONS}

mNGS is a useful diagnostic tool for non-HIVinfected PJP patients. It has a high sensitivity and specificity in identifying $P$. jirovecii and 
outperforms other methods in detecting mixed infections. mNGS of BALF and/or blood samples is suggested in patients with presumptive diagnosis of PJP. mNGS of blood samples may be a good alternative to BALF when bronchoscopic examination is infeasible.

\section{ACKNOWLEDGEMENTS}

The authors appreciate the assistance from Prof. Mingxiang Zou (Department of Clinical Laboratory, Xiangya Hospital, Central South University) and his colleagues. We acknowledge the professionalism and compassion demonstrated by all the healthcare workers involved in patient care. The authors thank all study participants for their involvement in the current study.

Funding. This work was supported by grants from the National Key R\&D Program of China (grant number 2016YFC1304204), Project Program of National Clinical Research Center for Geriatric Disorders (Xiangya Hospital, grant number 2020LNJJ05), National Natural Science Foundation of China (grant number 8160025), the Natural Science Foundation of Hunan Province (grant number S2019JJQNJJ1970) and the Youth Research Foundation of Xiangya Hospital (grant number 2018Q015). The Journal's Rapid Service Fee was funded by the authors.

Authorship. All named authors meet the International Committee of Medical Journal Editors (ICMJE) criteria for authorship for this article, take responsibility for the integrity of the work as a whole and have given their approval for this version to be published.

Author contributions. Yuanyuan $\mathrm{Li}$ and Pinhua Pan designed the study. Juan Jiang, Lu Bai, Yanhao Wu, Wenzhong Peng, Jian An and Wei Yang contributed to acquisition of data. Juan Jiang, Yuanyuan Li and Pinhua Pan analyzed the data. Juan Jiang wrote the draft of the paper. Yuanyuan Li and Pinhua Pan revised and edited the paper. All authors contributed to writing or reviewing the paper. All authors read and approved the final manuscript.
Disclosures. Juan Jiang, Lu Bai, Wei Yang, Wenzhong Peng, Jian An, Yanhao Wu, Pinhua Pan and Yuanyuan Li declare that they have no conflict of interest.

Compliance with ethics guidelines. This study was performed in accordance with the Helsinki Declaration of 1964 and its later amendments. Ethical approval for this study was obtained from the Institutional Review Board and Ethics Committee of Central South University. The ethics committee approved the waiver of informed consent owing to the retrospective nature of the review. All research data were de-identified and anonymously analyzed.

Data availability. The datasets generated and/or analyzed during the current study are available from the corresponding author on reasonable request.

Open Access. This article is licensed under a Creative Commons Attribution-NonCommercial 4.0 International License, which permits any non-commercial use, sharing, adaptation, distribution and reproduction in any medium or format, as long as you give appropriate credit to the original author(s) and the source, provide a link to the Creative Commons licence, and indicate if changes were made. The images or other third party material in this article are included in the article's Creative Commons licence, unless indicated otherwise in a credit line to the material. If material is not included in the article's Creative Commons licence and your intended use is not permitted by statutory regulation or exceeds the permitted use, you will need to obtain permission directly from the copyright holder. To view a copy of this licence, visit http://creativecommons.org/licenses/by$\mathrm{nc} / 4.0 /$.

\section{REFERENCES}

1. Ghembaza A, Vautier M, Cacoub P, Pourcher V, Saadoun D. Risk factors and prevention of Pneumocystis jirovecii pneumonia in patients with 
autoimmune and inflammatory diseases. Chest. 2020;158(6):2323-32.

2. Cilloniz C, Dominedo C, Alvarez-Martinez MJ, Moreno A, Garcia F, Torres A, et al. Pneumocystis pneumonia in the twenty-first century: HIV-infected versus HIV-uninfected patients. Expert Rev Anti Infect Ther. 2019;17(10):787-801.

3. Procop GW, Haddad S, Quinn J, Wilson ML, Henshaw NG, Reller LB, et al. Detection of Pneumocystis jiroveci in respiratory specimens by four staining methods. J Clin Microbiol. 2004;42(7):3333-5.

4. White PL, Backx M, Barnes RA. Diagnosis and management of Pneumocystis jirovecii infection. Expert Rev Anti Infect Ther. 2017;15(5):435-47.

5. Del Corpo O, Butler-Laporte G, Sheppard DC, Cheng MP, McDonald EG, Lee TC. Diagnostic accuracy of serum (1-3)-beta-D-glucan for Pneumocystis jirovecii pneumonia: a systematic review and meta-analysis. Clin Microbiol Infect. 2020;26(9): 1137-43.

6. Limper AH, Offord KP, Smith TF, Martin WJ II. Pneumocystis carinii pneumonia. Differences in lung parasite number and inflammation in patients with and without AIDS. Am Rev Respir Dis. 1989;140(5): 1204-9.

7. Rossen JWA, Friedrich AW, Moran-Gilad J, Genomic ESGF, Molecular D. Practical issues in implementing whole-genome-sequencing in routine diagnostic microbiology. Clin Microbiol Infect. 2018;24(4):355-60.

8. Han D, Li R, Shi J, Tan P, Zhang R, Li J. Liquid biopsy for infectious diseases: a focus on microbial cell-free DNA sequencing. Theranostics. 2020;10(12):5501-13.

9. Wu X, Li Y, Zhang M, Li M, Zhang R, Lu X, et al. Etiology of severe community-acquired pneumonia in adults based on metagenomic next-generation sequencing: a prospective multicenter study. Infect Dis Ther. 2020;9(4):1003-15.

10. Zhang Y, Ai JW, Cui P, Zhang WH, Wu HL, Ye MZ. A cluster of cases of pneumocystis pneumonia identified by shotgun metagenomics approach. J Infect. 2019;78(2):158-69.

11. Chen J, He T, Li X, Wang X, Peng L, Ma L. Metagenomic next-generation sequencing in diagnosis of a case of Pneumocystis jirovecii pneumonia in a kidney transplant recipient and literature review. Infect Drug Resist. 2020;13:2829-36.

12. Zhang K, Yu C, Li Y, Wang Y. Next-generation sequencing technology for detecting pulmonary fungal infection in bronchoalveolar lavage fluid of a patient with dermatomyositis: a case report and literature review. BMC Infect Dis. 2020;20(1):608.

13. Jeon YJ, Zhou Y, Li Y, Guo Q, Chen J, Quan S, et al. The feasibility study of non-invasive fetal trisomy 18 and 21 detection with semiconductor sequencing platform. PLoS One. 2014;9(10):e110240.

14. Schmieder R, Edwards R. Quality control and preprocessing of metagenomic datasets. Bioinformatics. 2011;27(6):863-4.

15. Li H, Durbin R. Fast and accurate long-read alignment with Burrows-Wheeler transform. Bioinformatics. 2010;26(5):589-95.

16. Quinlan AR, Hall IM. BEDTools: a flexible suite of utilities for comparing genomic features. Bioinformatics. 2010;26(6):841-2.

17. Peng JM, Du B, Qin HY, Wang Q, Shi Y. Metagenomic next-generation sequencing for the diagnosis of suspected pneumonia in immunocompromised patients. J Infect. 2021;82(4):22-7.

18. Gaborit BJ, Tessoulin B, Lavergne RA, Morio F, Sagan C, Canet E, et al. Outcome and prognostic factors of Pneumocystis jirovecii pneumonia in immunocompromised adults: a prospective observational study. Ann Intensive Care. 2019;9(1):131.

19. Maschmeyer G, Helweg-Larsen J, Pagano L, Robin C, Cordonnier C, Schellongowski P, et al. ECIL guidelines for treatment of Pneumocystis jirovecii pneumonia in non-HIV-infected haematology patients. J Antimicrob Chemother. 2016;71(9): 2405-13.

20. Wang J, Han Y, Feng J. Metagenomic next-generation sequencing for mixed pulmonary infection diagnosis. BMC Pulm Med. 2019;19(1):252.

21. Langelier C, Kalantar KL, Moazed F, Wilson MR, Crawford ED, Deiss $\mathrm{T}$, et al. Integrating host response and unbiased microbe detection for lower respiratory tract infection diagnosis in critically ill adults. Proc Natl Acad Sci USA. 2018;115(52): E12353-62.

22. Xie Y, Du J, Jin W, Teng X, Cheng R, Huang P, et al. Next generation sequencing for diagnosis of severe pneumonia: China, 2010-2018. J Infect. 2019;78(2):158-69.

23. Dubourg G, Raoult D. Emerging methodologies for pathogen identification in positive blood culture testing. Expert Rev Mol Diagn. 2016;16(1):97-111.

24. Ramachandran PS, Wilson MR. Metagenomics for neurological infections-expanding our imagination. Nat Rev Neurol. 2020;16(10):547-56. 
25. Thoendel MJ, Jeraldo PR, Greenwood-Quaintance $\mathrm{KE}$, Yao JZ, Chia N, Hanssen AD, et al. Identification of prosthetic joint infection pathogens using a shotgun metagenomics approach. Clin Infect Dis. 2018;67(9):1333-8.

26. Parize P, Muth E, Richaud C, Gratigny M, Pilmis B, Lamamy $A$, et al. Untargeted next-generation sequencing-based first-line diagnosis of infection in immunocompromised adults: a multicentre, blinded, prospective study. Clin Microbiol Infect. 2017;23(8):574 (e1-e6).

27. Pan T, Tan R, Qu H, Weng X, Liu Z, Li M, et al. Next-generation sequencing of the BALF in the diagnosis of community-acquired pneumonia in immunocompromised patients. J Infect. 2019;79(1):61-74.

28. Hammarstrom H, Grankvist A, Broman I, Kondori $\mathrm{N}$, Wenneras C, Gisslen $M$, et al. Serum-based diagnosis of Pneumocystis pneumonia by detection of Pneumocystis jirovecii DNA and 1,3-beta-D-glucan in HIV-infected patients: a retrospective case control study. BMC Infect Dis. 2019;19(1):658.

\section{Publisher's Note}

Springer Nature remains neutral with regard to jurisdictional claims in published maps and institutional affiliations. 